Published in "Soft Matter 9(32): 7843-7854, 2013"

which should be cited to refer to this work.

\title{
Star colloids in nematic liquid crystals
}

\begin{abstract}
Clayton P. Lapointe, ${ }^{\star a b c}$ Kenny Mayoral ${ }^{\mathrm{b}}$ and Thomas G. Mason*abd
We present a study of the elastic alignment, accompanying director field distortions, and elastic pair interactions of star-shaped colloids suspended in aligned nematic liquid crystals. We design and fabricate lithographic colloids, " $N$-stars", containing $N$ rod-like protrusions (i.e. "rays" or "arms") each having a constant angle between adjacent rays. $\mathrm{N}$-star geometries contain concave regions while retaining the rotational and mirror symmetries of regular polygonal platelets having $N$ sides. Planar anchoring of the nematic director at $\mathrm{N}$-star surfaces induces elastic deformations of the uniform background director, resulting in distinct orientational states and pair interactions that depend upon $N$. Director fields around isolated $\mathrm{N}$-stars are characterized using polarized optical microscopy. For each $\mathrm{N}$ star, we observe long-lived metastable orientational states with accompanying metastable director configurations, which are topologically distinct from the ground state director field. We develop a model, based on a superposition of the elastic energy of rod-like inclusions at appropriate angles to the far-field director, to estimate the energies in both cases. Numerical calculations of the director field around an individual ray elucidate the effect of azimuthal degeneracy in the anchoring and crosssectional shape of the ray. The analytical results agree with the simulations, however, we find that the total elastic energy must be rescaled to account for weaker anchoring. The long-range elastic pair interactions between $\mathrm{N}$-stars are probed using optical tweezers and video microscopy. We observe a distinct multipole depending on whether $N$ is even or odd, which dominates the distance-dependence for attractive elastic forces between pairs of $\mathrm{N}$-stars. Finally, we discuss assemblies made up of mixtures of different types of $\mathrm{N}$-stars that display a variety of aggregated states.
\end{abstract}

\section{Introduction}

Control over the detailed geometry, composition, and interfacial properties of colloidal particles is providing exciting opportunities for either their spontaneous or directed assembly, which may lead to new types of potentially useful soft materials. The advent of colloidal fabrication techniques that allow for the mass production of a large variety of colloidal shapes ${ }^{1-8}$ with high fidelity has led to a growing appreciation of the role that geometrical shape may play both fundamentally as well as in the design or exploitation of such interactions. ${ }^{8-19}$ For instance, micron-sized spherical colloids pinned to a fluid-fluid interface experience capillary forces mediated by surface tension, which can induce crystallization at a flat meniscus, ${ }^{\mathbf{2 0 , 2 1}}$ whereas elongated colloids at a curved interface experience aligning torques as well as forces..$^{22-24}$ In another important example, entropic depletion interactions mediated by a solution

${ }^{a}$ Department of Physics and Astronomy, University of California-Los Angeles, Los Angeles, CA 90095, USA. E-mail: clayton.lapointe@unifr.ch; mason@chem.ucla.edu ${ }^{b}$ Department of Chemistry and Biochemistry, University of California-Los Angeles, Los Angeles, CA 90095, USA

'Physics Department, University of Fribourg, Fribourg, CH-1700, Switzerland ${ }^{d}$ California NanoSystems Institute, University of California-Los Angeles, Los Angeles, CA 90095, USA of solvated polymers or smaller colloidal particles have been utilized to design and drive the assembly of a wide-range of colloidal aggregates having shape-selective structures. ${ }^{\mathbf{8}, 11-14,19}$

Along these lines, anisotropic colloidal interactions mediated by orientational elasticity within nematic liquid crystals (NLCs) have also received considerable attention..$^{15,25-59}$ Thermotropic NLCs are often composed of rod-shaped molecules that align parallel to one another while within the nematic phase, whereas at temperatures above the nematic-isotropic transition $T_{\mathrm{NI}}$ in the isotropic phase, they are randomly oriented. Micron-scale colloids suspended within NLCs deform the uniform alignment of the molecules due to the preferential alignment at the curved surfaces of the colloids (i.e. surface anchoring). The local alignment direction is often represented with a vector field, referred to as the director field, $\mathbf{n}(\mathbf{r})$, which has unit magnitude $(|\mathbf{n}(\mathbf{r})| \equiv 1)$, and must also be invariant with respect to an inversion, $\mathbf{n}(\mathbf{r}) \rightarrow-\mathbf{n}(\mathbf{r})$, due to the non-polar orientational ordering of NLCs. ${ }^{47}$ The ground state is uniform, i.e. $\mathbf{n}(\mathbf{r})=\mathbf{n}_{0}$, whereas introducing deformations of $\mathbf{n}(\mathbf{r})$ away from $\mathbf{n}_{0}$ cost elastic energy. These are known to engender strong, anisotropic interactions when the deformation fields of two or more colloids overlap. Analogous to the electrostatic forces that act between localized charge distributions, elastic colloidal interactions exhibit multipolar symmetries at long-range, such as dipolar and quadrupolar to lowest order..$^{25-28}$ 
For a colloidal particle of a given size, the particular $\mathbf{n}(\mathbf{r})$ configuration surrounding the particle can be modified through applying external fields, confining surfaces, as well as altering the interfacial surface anchoring properties of the colloid. ${ }^{30}$ In addition, for a particular type and strength of surface anchoring, $\mathbf{n}(\mathbf{r})$ surrounding isolated colloids, and consequently their elastic pair interactions, can be altered dramatically through a small change of the colloids' shape. This was demonstrated using convex regular polygonal colloids such as triangular, square-shaped, and pentagonal platelets that promote planar anchoring of $\mathbf{n}(\mathbf{r})$ with no in-plane azimuthal preference for $\mathbf{n}(\mathbf{r})$ at their surfaces,${ }^{15}$ an anchoring condition often referred to as planar degenerate anchoring. ${ }^{47}$ On the other hand, polymer colloidal spheres which display planar degenerate anchoring are known to only produce elastic quadrupoles with this type of anchoring. ${ }^{28,30,35}$

In this work, we study the shape-induced $\mathbf{n}(\mathbf{r})$ configurations and elastic pair interactions of lithographically fabricated starshaped colloids, which we refer to as $N$-stars. Their geometries were specifically designed to contain regions of concavity while having the same mirror and rotational symmetries as regular convex polygons. We characterize $\mathbf{n}(\mathbf{r})$ surrounding isolated $N$ stars suspended in aligned NLCs using polarized optical microscopy. Interestingly, $N$-stars readily form metastable director configurations, which have not been observed for other shapes such as regular polygons. Using a combination of analytical and numerical techniques, we develop a simple model in which the elastic energy of an $\mathrm{N}$-star as a function of its orientation can be approximated as a superposition of rods held at appropriate angles to the NLC's far-field alignment direction. In addition, we characterize their pair interactions using optical tweezers and particle tracking video microscopy. At large separations, a strong, elastic orientational potential constrains the orientation of each $\mathrm{N}$-star, whereas at shortrange, near-field elasticity can induce significant body rotations via elastic torques. This near-field interaction affects the detailed structure of two $\mathrm{N}$-stars near contact. Consequently, the final colloidal aggregate's structure depends on both the nature of the long-range pair interaction as well as short-range effects when two $N$-stars are close to one another.

\section{Experimental}

\subsection{Lithographic $N$-star colloid fabrication and dispersion in $5 \mathrm{CB}$}

We fabricate three different star-shaped particles (i.e. " $N$-stars") having $N=3,4$, or 5 rod-like rays joined to a central core, where $\alpha_{N}=2 \pi / N$ are the angles between adjacent rays. Within a $N$-star, each ray is $L=3.0 \mu \mathrm{m}$ in length and has a rectangular crosssection having width $w=0.4 \mu \mathrm{m}$ and height $h=1.0 \mu \mathrm{m}$ set by the thickness of the resist layer during fabrication. The central core geometry that connects the $N$ rays was designed to be a regular polygon having $N$ in-plane sides of $0.4 \mu \mathrm{m}$, corresponding to the designed width $w$ of the rays. For all three $N$-stars, the largest difference from the center to the end of an ray is very small, $\approx 50 \mathrm{~nm}$, well below the $\approx 300 \mathrm{~nm}$ feature size of our lithography system and the resolution of our optical microscope. ${ }^{7}$ Also, the fabrication process results in some rounding of corners and edges of the printed features; radii of curvatures are typically in the range from about 100 to $200 \mathrm{~nm}$ depending on the opening angle of the corner and the distance between adjacent corners. Different than regular polygons, which contain convex curvature concentrated at $N$ vertices, a key feature of $N$-star geometries is the presence of concave curvature at $(N-1)$ locations where adjacent rays meet near the core in addition to convex curvature at the rays' ends.

Briefly, we describe our protocol used to lithographically fabricate star colloids and transfer them from an organic solvent to a NLC. ${ }^{15}$ First, a release layer consisting of a $28 \mathrm{wt} \%$ solution of SU-8 resin in cyclopentanone (Sigma-Aldrich) is spin-coated onto a 5 inch Si wafer and soft baked resulting in a $1.0 \mu \mathrm{m}$ thickness. A second $1.0 \mu \mathrm{m}$ layer of SU-8 2001 (Microchem) resist is spun onto the release layer, soft baked, and then exposed in a 5:1 reduction i-line stepper (Ultratech). After a post exposure bake, the wafers are immersed in SU-8 developer, 1-methoxy-2-propyl acetate (PGMEA), and agitated in an ultrasonic bath, lifting off the cross-linked particles into the developer. Dissolved SU-8 resin was removed from the suspension by repeated centrifugation, decanting, and washing with fresh PGMEA. A small volume of $N$-star/PGMEA dispersion $(\approx 200 \mu \mathrm{L})$ was added to high-purity pentylcyano-biphenyl (5CB) at a $1: 1$ ratio by volume, mixed thoroughly, and placed in a convection oven evacuated with a rotary pump and held at a temperature $T=60{ }^{\circ} \mathrm{C}$ for at least 24 hours. The low vapor pressure of $5 \mathrm{CB}$ $\left(\approx 0.2 \mathrm{~Pa} \text { at } T=90^{\circ} \mathrm{C}\right)^{60}$ and high solubility with PGMEA allows for the removal of residual PGMEA under vacuum.

Single-crystal nematic cells were assembled using two glass plates coated with thin layers of rubbed polyimide (PI-2555, HD Microsystems). Polyimide anchors $\mathbf{n}(\mathbf{r})$ parallel to the rubbing direction with a small out of plane tilt angle $\approx 1-2^{\circ}$. The rubbing directions at the opposing alignment layers were oriented anti-parallel so that $\mathbf{n}(\mathbf{r})$ was uniform throughout the bulk and defined the direction of the far-field director $\mathbf{n}_{0}$. Monodisperse glass beads having $10 \mu \mathrm{m}$ diameters served as spacers to define the cell gap. Sample cells were filled with $\mathrm{N}$ star/5CB dispersions by capillary action such that the air-5CB interface traveled either predominately parallel to or perpendicular to the rubbing direction while $5 \mathrm{CB}$ was in the nematic phase. Volume fractions of $N$-star/5CB dispersions were kept dilute $\left(\sim 10^{-5}\right.$ to $\left.10^{-4}\right)$ so that individual particles and isolated pairs of particles could be readily studied.

\subsection{Optical tweezers}

We use single beam gradient optical tweezers based on a $647 \mathrm{~nm}$ diode laser (Toptica iBeam-Smart-640.5) and an inverted microscope (Nikon Ti-Eclipse/A1R-MP) to assemble collections of $\mathrm{N}$-stars for interaction studies. Since the beam mode from the diode is of low quality, we first spatially filter the beam using a polarization preserving single mode optical fiber. An airspaced doublet collimator attached to the fiber expands the beam to a size that slightly overfills the back aperture of the microscope objective. A rotatable half-wave plate is used to control the orientation of the linear polarization state of the 
laser. A dichroic mirror directs the beam towards either a $40 \times$ (Nikon Plan Fluor $40 \times$ Oil, NA $=1.3$ ) or a $100 \times$ objective (Nikon Apo $100 \times$ TIRF, NA = 1.49), which are used to both focus the laser and image the focal plane. A CCD camera (Andor iXon+) is used to obtain digital video of the field of view.

The laser polarization is oriented perpendicular to $\mathbf{n}_{0}$ so that the uniform $\mathbf{n}(\mathbf{r})=\mathbf{n}_{0}$ away from a $N$-star presents an effective refractive index $n_{\text {eff }}=n_{\perp}=1.5$. Regions of an $N$-star having a refractive index $n_{\text {SU-8 }} \approx 1.6$ as well as the locations near the rays of a star with significant $\mathbf{n}(\mathbf{r})$ distortions $\left(1.5 \leq n_{\text {eff }} \leq 1.7\right)$ are drawn into the focus of an optical tweezer since both $n_{\text {SU- } 8}$ and $n_{\text {eff }}$ are greater than $n_{\perp} \cdot{ }^{15,61-63}$ By moving the trap away from a specific ray using a translation stage, $N$-stars can be dragged at average speeds up to roughly $2 \mu \mathrm{m} \mathrm{s}^{-1}$. Raising the optical power above $\approx 15 \mathrm{~mW}$ results in a distorted spot at the focus due to the optical Fréedericksz effect. ${ }^{62-64}$ The optical field interacts with the local dielectric anisotropy of the NLC and torques $\mathbf{n}(\mathbf{r})$ away from $\mathbf{n}_{0}$. In this case, selective rays of the $\mathrm{N}$ stars are attracted to the spot with a different overall force depending on the sense of the $\mathbf{n}(\mathbf{r})$ rotation relative to that of a specific ray.

To study elastic pair interactions, we position isolated pairs of $\mathrm{N}$-stars to prescribed locations and track their subsequent motion under elastic forces using video microscopy. Digital videos are stored on a computer and image analysis routines are used to measure the positions of both $\mathrm{N}$-stars within each frame in order to construct trajectories. Due to the complexity of their shapes, significant rotations of $N$-stars about $\mathbf{n}_{0}$ result in complicated brightfield images that are difficult to deconvolve using image analysis. We mitigate this by using cells with gaps $(D \approx 10 \mu \mathrm{m})$ somewhat larger than the lateral size of the $N$-stars $(l \approx 5.6 \mu \mathrm{m})$. When $D \sim l$, the elastic repulsion between each ray from the top and bottom alignment layers constrains rotations of the $N$-stars about $\mathbf{n}_{0}$ so that they lie with all of their rays nearly parallel to the glass substrates. This facilitates quantitative tracking of the $\mathrm{N}$-stars' motion using video microscopy over longer time periods ( $\sim$ hours) than can be accomplished in cells having larger gaps $(\sim 1-10 \mathrm{~s})$. Although this method works for isolated $\mathrm{N}$-stars, when two or more $\mathrm{N}$-stars are within a distance $\sim D$ of each other, in many cases, these nearby $N$-stars rotate about $\mathbf{n}_{0}$ to alleviate distortions in the director field and reduce elastic energy.

\section{Results and discussion}

\subsection{Shape-dependent elastic alignment and symmetry of director deformations}

Shown in Fig. 1 are microscopy images of $N$-stars in their respective ground state configurations suspended in a uniformly aligned nematic 5CB. As can be seen in Fig. 1a, 3stars align such that one ray is parallel to $\mathbf{n}_{0}$ with no accompanying distortions due to the planar surface anchoring of $\mathbf{n}(\mathbf{r})$ on SU-8, and the two adjacent rays oriented at $30^{\circ}$ to $\mathbf{n}_{0}$ are surrounded by bright textures. Fig. 1d shows a microscopy image taken under parallel polarizers with their transmission axes parallel to $\mathbf{n}_{0}$. In Fig. $1 \mathrm{~g}$, we schematically depict $\mathbf{n}(\mathbf{r})$
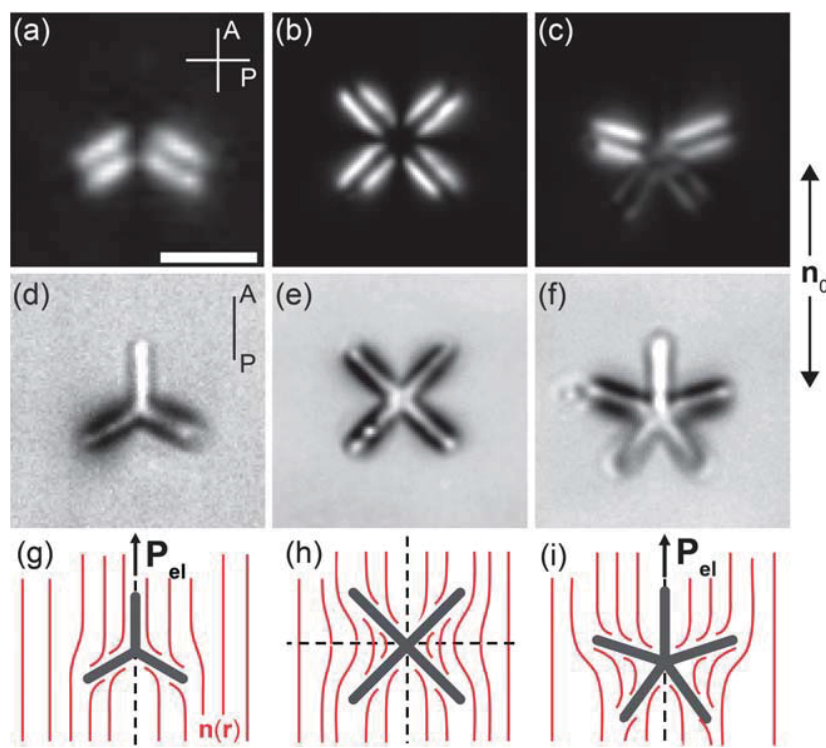

(e)

(f)
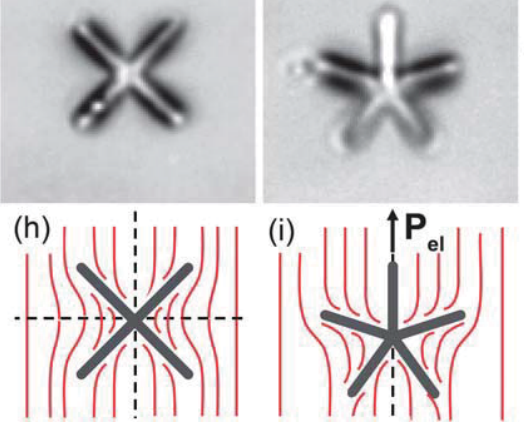

Fig. 1 Optical microscopy images under crossed polarizers (a-c) and paralle polarizers ( $d-f)$ showing isolated $N$-star colloids in their respective ground states. $N$-stars having odd $N$, such as (a and d) 3-stars and (c and f) 5-stars, align in the ground state with one ray parallel to $\mathbf{n}_{0}$ and give rise to elastic dipoles with $\mathbf{p}_{\mathrm{el}} \|$ $\mathbf{n}_{0}$. 4-stars having even $N$ give rise to quadrupolar $\mathbf{n}(\mathbf{r})$ deformations in the ground state and align such that all rays are at $45^{\circ}$ to $\mathbf{n}_{0}(\mathrm{~b}$ and e). ( $\mathrm{g}-\mathrm{i})$ Schematics of the ground state director field $\mathbf{n}(\mathbf{r})$ (red curves) for each $N$-star. Dashed lines indicate mirror symmetry planes of $\mathbf{n}(\mathbf{r})$. Scale bar: $5 \mu \mathrm{m}$.

surrounding the 3-star inferred from images such as the ones shown in Fig. 1a and d.

Both 3-stars and 5-stars give rise to dipolar $\mathbf{n}(\mathbf{r})$ with one ray parallel to $\mathbf{n}_{0}$, whereas 4 -stars induce quadrupolar $\mathbf{n}(\mathbf{r})$ deformations with all four rays oriented at $45^{\circ}$ to $\mathbf{n}_{0}$ as shown schematically in Fig. 1g-i. These ground state orientations can be understood by considering the particle's shape, the planar surface anchoring boundary conditions at the colloid's edges, and the possible orientations which give at the very least dipolar symmetry [i.e. at least one horizontal and one vertical mirror symmetry plane for $\mathbf{n}(\mathbf{r})] \cdot{ }^{15,46-51}$ All orientations for which all planes of $\mathbf{n}(\mathbf{r})$ 's mirror symmetry are broken requires an external torque to act on the fluid. ${ }^{46-48}$ Therefore, this set of orientations are mechanically unstable, since an elastic torque will tend to rotate the colloid such that at least one vertical plane of mirror symmetry exists, leaving only either dipolar or quadrupolar configurations as possibilities. This process must occur after the 5CB solvent is thermally quenched from the isotropic phase to the nematic phase during sample preparation, and it has been directly observed using SU-8 triangular platelets in $5 \mathrm{CB} .{ }^{15}$

Similar to $\mathrm{N}$-sided regular polygonal platelets, $\mathrm{N}$-star geometrical shapes exhibit $N$-fold rotational symmetry about the axis passing through the center of the $N$-star and orthogonal to all rays. Considering $N$-star geometry in two-dimensions as depicted in the bottom rows of Fig. 1 and 2, each $N$-star displays $N$ mirror planes parallel to and passing through each of the $N$ rays. In the presence of a NLC, the dominant multipole of $\mathbf{n}(\mathbf{r})$ deformations, either dipolar or quadrupolar, of both the ground and metastable configurations follow from these reflection 

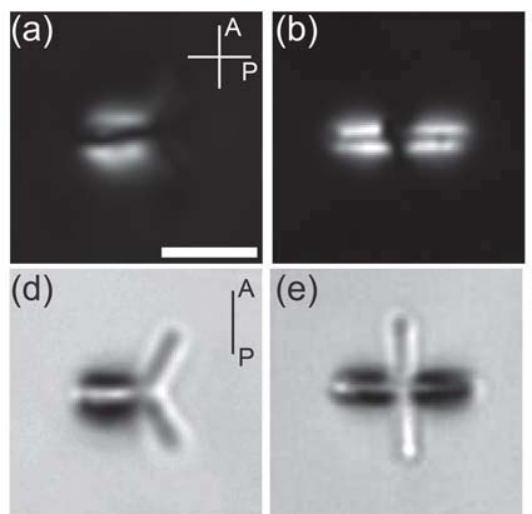

(e)

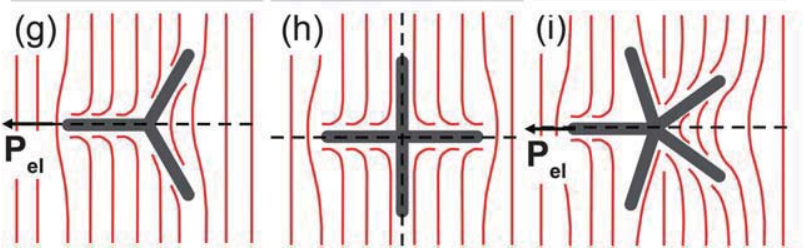

Fig. 2 Optical microscopy images showing metastable configurations under crossed polarizers $(a-c)$ and parallel polarizers $(d-f)$. Metastable states for odd $N$ [e.g. 3-stars ( $\mathrm{a}, \mathrm{d}$ ) and 5-stars ( $\mathrm{c}$ and f)] align with one ray perpendicular to $\mathbf{n}_{0}$ and give rise to elastic dipoles with $\mathbf{p}_{\mathrm{el}} \perp \mathbf{n}_{0}$. Metastable 4-stars (b and e) are elastic quadrupoles and orient such that two rays are at $90^{\circ}$ to $\mathbf{n}_{0}$. (g-i) Schematics of the director field $\mathbf{n}(\mathbf{r})$ (red curves) surrounding each type of $N$-star. Dashed lines indicate mirror symmetry planes of $\mathbf{n}(\mathbf{r})$. Scale bar: $5 \mu \mathrm{m}$.

symmetries. $N$-stars having odd $N$ such as 3 -stars lack fore-aft reflection symmetry, and therefore, give rise to dipolar $\mathbf{n}(\mathbf{r})$, whereas 4-stars possess this symmetry and quadrupolar configurations are stable. Following refs. 51 and 54 we refer to these mirror planes as "vertical". In three-dimensions, for each $\mathrm{N}$-star, there is an additional mirror plane passing through the mid-plane of the particle's thickness. In the subsequent discussion we omit this mirror plane from the analysis and consider a two-dimensional system - the mid-plane of the $\mathrm{N}$ star - since only the mirror symmetry of vertical planes are broken in our experiments.

Orienting a 3-star such that one ray is parallel to $\mathbf{n}_{0}$ along with planar anchoring boundary conditions would give a mechanically stable $\mathbf{n}(\mathbf{r})$ configuration similar to the one depicted in Fig. 1g. The deformations are dipolar with the elastic dipole moment $\mathbf{p}_{\mathrm{el}} \| \mathbf{n}_{0}$ since the normal of the vertical plane of mirror symmetry that is broken is parallel to $\mathbf{n}_{0}$. Another possible ground state orientation for a 3-star would be the configuration shown in Fig. 2g. The director field in this case has a broken-vertical mirror plane with its normal perpendicular to $\mathbf{n}_{0}$, and therefore, $\mathbf{p}_{\mathrm{el}} \perp \mathbf{n}_{0}$. Any other intermediate orientation will give rise to $\mathbf{n}(\mathbf{r})$ deformations that break all planes of mirror symmetry, and must be mechanically unstable. The same argument applies to $N$-stars having any $N$, with the caveat that for even $N$, the equilibrium states have quadrupolar symmetry as shown for a 4-star colloid in Fig. 1h, and dipolar symmetry for odd $N$, as is the case for 3-stars and 5-stars shown in Fig. $1 \mathrm{~g}$ and i respectively.

Just as regular polygons give rise to elastic dipoles for odd $N$ and quadrupoles for even $N,{ }^{15} \mathrm{~N}$-stars also induce elastic quadrupoles if $N$ is even and dipoles if $N$ is odd. However, beyond this qualitative similarity, there are important differences between the behavior of $N$-stars and regular polygons. By contrast to regular polygons, $N$-stars with odd $N$ rays induce an elastic dipole moment $\mathbf{p}_{\mathrm{el}}$ parallel to $\mathbf{n}_{0}$ in equilibrium whereas odd $N$ regular polygons induce $\mathbf{p}_{\mathrm{el}}$ orthogonal to $\mathbf{n}_{0}$. Furthermore, for both $N$-stars having odd $N$ studied in this work, in addition to an equilibrium ground state in which $\mathbf{p}_{\mathrm{el}}$ is parallel to $\mathbf{n}_{0}$, a higher energy metastable state wherein $\mathbf{p}_{\mathrm{el}}$ is orthogonal to $\mathbf{n}_{0}$ is also observed. For the even $N$ case (e.g. 4-stars), the metastable $\mathbf{n}(\mathbf{r})$ configuration is also quadrupolar; however, the $N$-star orientation is rotated by $\pi / 4$ relative to the ground state orientation. For the two dipolar metastable configurations, $\mathrm{N}$ star body frame orientations are shifted by an angle $\alpha_{N} / 4$ (e.g. $\pi / 6$ and $\pi / 10$ for 3 -stars and 5 -stars, respectively) relative to the ground state orientation and $\alpha_{N} / 2$ for the quadrupolar $N=4$ case. Moreover, the same degree of mirror symmetry of $\mathbf{n}(\mathbf{r})$ is preserved when comparing the ground and metastable $\mathbf{n}(\mathbf{r})$ for a given $N$.

For a particular $N$-star, of the two possible orientational states and accompanying $\mathbf{n}(\mathbf{r})$, the ground state corresponds to the one that costs less total elastic energy. We have identified the states shown in Fig. 1 as the lowest energy configurations in light of their much higher prevalence over the states shown in Fig. 2. We speculate that the metastable configurations could be induced by strong shear flows while filling the sample cell as $5 \mathrm{CB}$ is within the nematic phase or they may arise when the dispersion is initially quenched to the nematic phase. Typically, if a sample cell is filled such that the capillary flow is predominantly perpendicular to the rubbing direction, we find that anywhere from 0 to $5 \%$ of $N$-stars are in their respective metastable states after the flow has ceased, whereas the probability of observing such metastable states is much less when filling the cell parallel to the rubbing direction. These higher energy metastable states remain stable over long periods of time, indicating that there is a large energetic barrier to transition via a mechanical rotation of the $N$-star to the ground state orientation.

\subsection{Model for elastic orientational potential}

We estimate the elastic energies corresponding to the observed equilibrium ground states and metastable orientational states of $\mathrm{N}$-stars in 5CB using a model in which the $\mathbf{n}(\mathbf{r})$ deformations for a given state are represented as a superposition of rod-like inclusions held at the same orientations relative to $\mathbf{n}_{0}$ as the rays of the $N$-star. For planar anchoring of $\mathbf{n}(\mathbf{r})$ along the longaxis of the rod and making the approximation that the three independent elastic constants of the NLC are equal to their average value, the elastic energy $U_{\mathrm{el}}$ as a function of the angle between the long-axis of the rod and $\mathbf{n}_{0}, \phi$, can be expressed as, $U_{\mathrm{el}}(\phi)=2 \pi K C \phi^{2}$, where $K$ is an average elastic constant of the NLC and $C$ is the effective capacitance of the rod in units of length. ${ }^{46,56}$ In general, the effective capacitance depends on the dimensions of the rod as well as the distance between the rod and the confining walls..$^{5,56}$ 
Using $N$ rods to represent the $N$ rays of a given $N$-star, we label each ray with an index $i$, and estimate the total elastic energy as a sum:

$$
U_{\mathrm{G}, \mathrm{M}}=2 \pi K C \sum_{i=1}^{N} \phi_{i}{ }^{2},
$$

where $\phi_{i}$ is the angle between the long-axis of ray $i$ and $\mathbf{n}_{0}$ as defined in Fig. 3, and the subscripts $\mathrm{G}$ and $\mathrm{M}$ refer to the ground and metastable state, respectively. Using the observed orientations of both ground and metastable states for all three $N$-stars, we calculate the elastic energy scaled by $2 \pi K C, u_{\mathrm{G}, \mathrm{M}}=U_{\mathrm{G}, \mathrm{M}} /$ $(2 \pi K C)=\sum \phi_{i}{ }^{2}$, for all six observed states and tabulate them in Fig. 3. Note that $u_{\mathrm{G}, \mathrm{M}}$ is unitless since $K C$ has units of energy. Also included in Fig. 3 are estimates for the elastic energies $U_{\mathrm{G}, \mathrm{M}}$ in SI units using an expression for the capacitance $C$ of an individual ray valid for high aspect ratio cylinders, ${ }^{66} C \approx$ $L /[\ln (2 L / a)] \approx 1.6 \mu \mathrm{m}$, where $L=3 \mu \mathrm{m}$ and the cylinder radius $a=h=1 \mu \mathrm{m}$. For all $N$-stars, the scaled elastic energy of the metastable state $u_{\mathrm{M}}$ is always greater than that of the ground state $u_{\mathrm{G}}$ within this model. The difference $\Delta u=u_{\mathrm{M}}-u_{\mathrm{G}}$ is greatest for 4 -stars $\Delta u \approx 2.47$, while for 5-stars the difference is less $\Delta u \approx 0.49$, which may result from the increasing symmetry of $N$-star shapes with increasing $N$. These results are in qualitative agreement with the experimentally observed relative probabilities of such states. In our experiments, the presence of metastable 5-stars is much more likely than 4-stars.

The model includes a number of approximations: (1) elastic interactions between the rays are neglected, (2) the elastic energy arising from deformations near the central core is not included, (3) the one elastic constant approximation, and (4) the analytical result in eqn (1) is valid for uniform longitudinal anchoring over the entire surface of the ray. ${ }^{46}$ For many nematic liquid crystals, anisotropy of the three independent elastic constants is significant at temperatures far from $T_{\mathrm{NI}}$ (e.g. for $5 \mathrm{CB}$ at room temperature: $K_{11} / K_{33} \approx 5.8 \mathrm{pN} / 8 \mathrm{pN} \approx 0.7$ where $K_{11}$ and $K_{33}$ are the elastic constants associated with splay and bend deformations, respectively). ${ }^{65}$ However, including anisotropy in the elastic constants would only change $u_{\mathrm{G}}$ and $u_{\mathrm{M}}$ a comparable amount, assuming that no qualitative features of $\mathbf{n}(\mathbf{r})$ are altered significantly. Indeed, the director fields we observe by direct imaging of the ground and metastable state $\mathbf{n}(\mathbf{r})$ for any $N$-star appear qualitatively similar. Quantitative predictions of the effects due to (1) and (3) would require accurate three-dimensional numerical calculations that capture the complicated geometry of $\mathrm{N}$-stars and the resulting complex boundary conditions for $\mathbf{n}(\mathbf{r})$. We address (4) in Section 3.3 where results for the elastic energy of a rectangular crosssection ray that agree quantitatively with eqn (1) are discussed.

For each configuration, the stiffness $k$ of the orientational potential well, $U_{\mathrm{el}}(\theta)=k \theta^{2}$, can be determined by considering the change in the total elastic energy for a set of $N$ rays rotated by an angle $\theta$ as depicted for a 3-star in the ground state in Fig. 4a. Using eqn (1), and keeping track of the sense of the rotations (e.g. $\phi_{i} \rightarrow \phi_{i} \pm \theta$, where $\phi_{i} \rightarrow \phi_{i}+\theta$, if the $i$-th ray's angle relative to $\mathbf{n}_{0}$ increases after the whole $N$-star is rotated

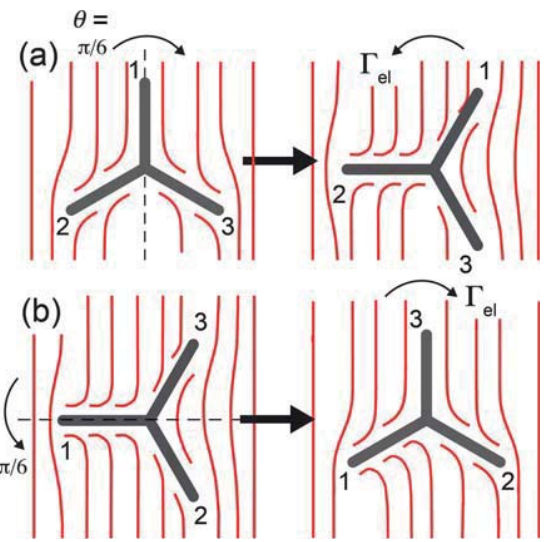

Fig. 4 (a) (Left) A 3-star in the ground state configuration. Numbers refer to the index $i$ used to label each ray and dashed lines denote mirror symmetry planes of $\mathbf{n}(\mathbf{r})$ (red curves). (Right) Following a clockwise rotation by $\theta=\pi / 6$ about an axis perpendicular to $\mathbf{n}_{0}$, all planes of mirror symmetry are broken, and the sense of the restoring elastic torque $\Gamma_{\mathrm{el}}$ is shown with the arrow. The sense of the $\mathbf{n}(\mathbf{r})$ curvature changes when crossing ray 2 , which is different than the symmetric $\mathbf{n}(\mathbf{r})$ surrounding the corresponding ray 1 in the metastable configuration as shown on the left of (b). Rotating a metastable 3-star by $\theta=\pi / 6$ counter-clockwise results in the $\mathbf{n}(\mathbf{r})$ shown on the right. Similar to the rotated ground state 3-star shown in the right hand side of (a), $\mathbf{n}(\mathbf{r})$ has no planes of mirror symmetry and a restoring torque acts with opposite sense to the imposed rotation. A defect would be necessary to transition from the $\mathbf{n}(\mathbf{r})$ shown on right side of (b) to that on the left side of (a). (a)
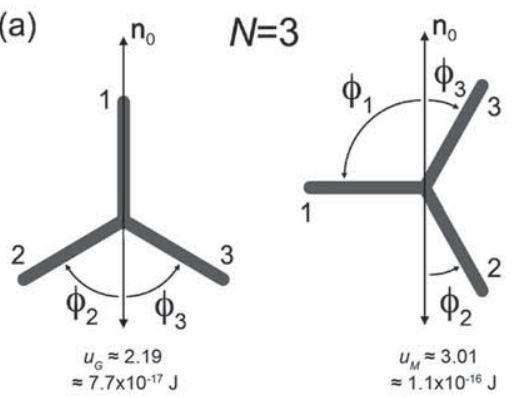
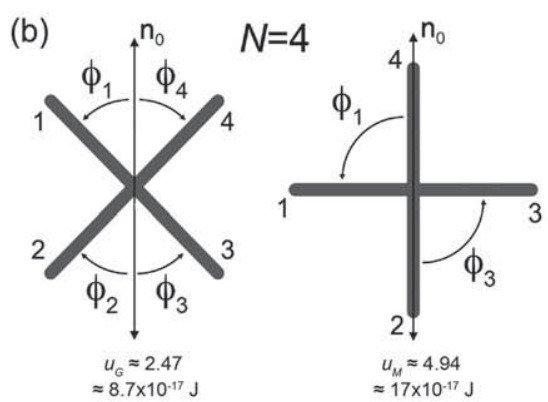
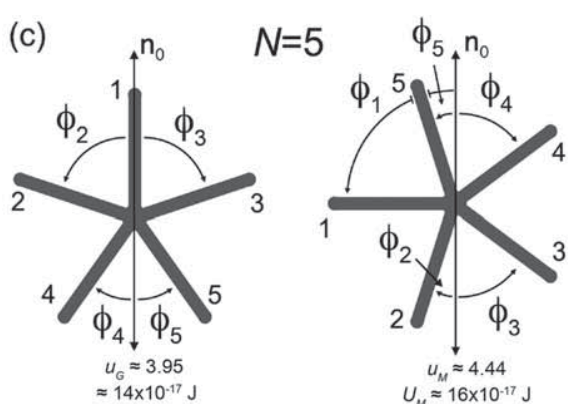

Fig. 3 Schematics showing the definition of the angles between each individual ray and the far-field director $\mathbf{n}_{0}$, $\phi_{i}$, for both the ground state (left) and metastable state (right) of each $N$-star: (a) $N=3$, (b) $N=4$, and (c) $N=5$. Values for the scaled elastic energies, $u_{\mathrm{G}}$ and $u_{\mathrm{M}}$, along estimates for the energies in real units are tabulated below each schematic as discussed in the text. 
clockwise), we find $U_{\mathrm{el}} \propto N \theta^{2}$, and the stiffness $k$ scales linearly with $N$. For example, considering a 3 -star in the ground state rotated by an angle $\theta$ clockwise about an axis perpendicular to $\mathbf{n}_{0}$, the sum $\sum \phi_{i}{ }^{2}$ in eqn (1) is $\sum \phi_{i}{ }^{2}=3 \theta^{2}+2 \pi^{2} / 9$. Including real units, this potential can be written $U_{\mathrm{el}}(\theta)=6 \pi K C \theta^{2}+4 \pi^{3} K C / 9=$ $6 \pi K C \theta^{2}+U_{0}$, where $U_{0}=4 \pi^{3} K C / 9$ is the equilibrium elastic energy at $\theta=0$. Rotations past $\pi / 2$ (i.e. for $\theta>\pi / 2$ ) should in principle result in the same energy as a corresponding rotation $\theta \rightarrow \theta-\pi / 2$, because of the inversion symmetry of the director field. ${ }^{46}$ However, experiments and simulations on cylindrical particles in 5 CB have shown that rotations past $\pi / 2$ result in metastable director configurations all the way up to $\theta \approx \pi .^{55,66}$

Observations have shown that $N$-star metastable states are stable over long time periods ( $\sim$ months) if the sample is stored without ever heating and melting the NLC solvent into the isotropic phase. The long-lived stability of the metastable configurations arises from a topological mismatch between the director fields associated with the ground and metastable states. In Fig. 4a, schematic drawings of $\mathbf{n}(\mathbf{r})$ show the sense of $\mathbf{n}(\mathbf{r})$ curvature after rotating the 3 -star by $30^{\circ}$. Comparing $\mathbf{n}(\mathbf{r})$ for the metastable configuration on the left hand side of Fig. $4 \mathrm{~b}$ to $\mathbf{n}(\mathbf{r})$ on the right of Fig. $4 \mathrm{a}$ for the rotated ground state shows that the director field cannot transition to the ground state $\mathbf{n}(\mathbf{r})$ without introducing a defect and/or possibly rotating out-ofplane. Furthermore, the sense of the restoring elastic torque in both cases opposes the imposed rotation and tends to rotate the 3-star back to its original $\mathbf{n}(\mathbf{r})$ configuration. In addition to this topological barrier, there is an elastic potential energy cost that constrains thermally-activated rotations to be small at all timescales. We typically measure orientational fluctuations that are on average less than $1^{\circ}$ at room temperature. Again estimating the order of magnitude capacitance of a singe ray using an expression valid for a high aspect ratio cylinder, ${ }^{66} C \sim L / \ln (2 L / a)$ $\sim 1 \mu \mathrm{m}$, where the ray's length and radius are $L=3 \mu \mathrm{m}$ and $a=$ $h=1 \mu \mathrm{m}$, respectively. Using a representative value for the average elastic constant of $5 \mathrm{CB}, K=4 \mathrm{pN}$ at $T=23{ }^{\circ} \mathrm{C},{ }^{65}$ the stiffness for the metastable branch is of order $k \sim 10^{-17} \mathrm{~J} \mathrm{rad}^{-2}$. Rotating a metastable 3 -star by $30^{\circ}$ so that it matches the orientation of a ground state 3-star would cost $\Delta U_{\mathrm{el}} \sim 10^{-18} \mathrm{~J}$ which is $\sim 10^{3}$ times larger than $k_{\mathrm{B}} T$ at ambient temperatures. We note that if $\mathbf{n}(\mathbf{r})$ singularities such as line disclinations or point defects are necessary to transition from the metastable branch to the ground state branch, as is the case for externally torqued axially-symmetric bodies such as cylinders ${ }^{66}$ and disks ${ }^{59}$ in NLCs, then the appropriate core energies of the defects would also contribute to $\Delta U_{\mathrm{el}}$.

\subsection{Numerical calculation of the director field near a ray}

In our model of the orientational elastic potential of $\mathrm{N}$-stars, we use the harmonic, $\phi^{2}$ dependence of the elastic free energy of single rays to calculate the total elastic energies of various orientational states for different $N$. The result is valid, within the one elastic constant approximation, for large $\phi(\phi \sim 1)$, and has been derived analytically for the case of arbitrarily shaped bodies with strong planar anchoring of $\mathbf{n}(\mathbf{r})$ within an infinite NLC using an analogy to the electrostatic problem of a charged conductor held at fixed voltage in infinite free space. ${ }^{46}$ Lattice Boltzmann simulations of this problem, which included anisotropy in the elastic constants, showed slight deviations from a harmonic potential. ${ }^{66}$ The presence of a wall that promotes strong, planar anchoring allows for elastic forces in addition to torques, yet the $\phi^{2}$ dependence of the energy still holds. ${ }^{55,68}$ However, as detailed Monte Carlo and molecular dynamics simulations of cylinders with finite homeotropic anchoring have shown, the elastic orientational potential of a non-spherical body is not always harmonic and can depend upon the locations of bulk defects as well as the finite nature of the surface anchoring strength. ${ }^{71}$ Analytical work also suggests that surface anchoring type and strength can be a crucial factor in these problems. ${ }^{69,70}$

In this section, we calculate numerically the elastic free energy of a ray held at various angles $\phi$ to $\mathbf{n}_{0}$ using a realistic geometry as well as more reasonable boundary conditions for $\mathbf{n}(\mathbf{r})$, and verify that the energy is indeed harmonic to large $\phi$. The elastic free energy as a function $\phi$ is calculated, and the result is used to determine explicit values for the capacitance per unit length of a single ray. In particular, we consider a rectangular cross-sectional rod having width $w=0.5 \mu \mathrm{m}$ and height $h=1 \mu \mathrm{m}$ immersed in an NLC with the long-axis of the rod held at an angle $\phi$ relative to the anchoring direction on two adjacent plates $5 \mu \mathrm{m}$ above and below the rod (i.e. cell gap, $D=10 \mu \mathrm{m}$ ). We define the long-axis of the ray to lie parallel to the planes of the bounding walls as well as the $x$-axis of a right-handed Cartesian coordinate system with the origin at the center of the ray. Since the long-axis of the rod lies in a plane parallel to the bounding walls and the anchoring is planar, we make the assumption that there is no tilt of the director out of the $x-y$ plane and express the director field as $\mathbf{n}(\mathbf{r})=\cos (\alpha(y, z)) \hat{\boldsymbol{x}}+\sin (\alpha(y, z)) \hat{\boldsymbol{y}}$ where $\alpha(y, z)$ is the local angle between $\mathbf{n}(\mathbf{r})$ and the $x$-axis.

The elastic free energy arising from spatial gradients of $\mathbf{n}(\mathbf{r})$ can be expressed using the Frank-Oseen free energy, which can be written as

$$
U_{\mathrm{el}}=\frac{1}{2} \int \mathrm{d}^{3} r\left[K_{1}(\nabla \cdot \mathbf{n})^{2}+K_{2}(\mathbf{n} \cdot \nabla \times \mathbf{n})^{2}+K_{3}(\mathbf{n} \times \nabla \times \mathbf{n})^{2}\right],
$$

where $K_{1}, K_{2}$, and $K_{3}$ are elastic constants for splay, twist and bend deformations of $\mathbf{n}(\mathbf{r})$, respectively. ${ }^{47}$ Setting all three elastic constants equal to their average value $K$ simplifies the integrand in eqn (2) to $(K / 2)\left[(\boldsymbol{\nabla} \cdot \mathbf{n})^{2}+(\boldsymbol{\nabla} \times \mathbf{n})^{2}\right]$. In terms of $\alpha(y, z)$, eqn (2) simplifies to

$$
U_{\mathrm{el}}=\frac{K}{2} \int \mathrm{d}^{3} r(\nabla \alpha)^{2}
$$

Minimizing $U_{\mathrm{el}}$ with respect to the functional $\alpha$ gives Laplace's equation

$$
\nabla^{2} \alpha=0
$$

We obtain numerical solutions to eqn (4) within the $y-z$ plane, $\alpha(y, z)$, using a relaxation method on a 300 by 200 square grid. To obtain reasonable spatial accuracy near the edges of the ray where large gradients of $\alpha$ are expected, the 
grid spacing is defined to be $1 / 10$ of the width of a ray $w=0.5$ $\mu \mathrm{m}$. Periodic boundary conditions are imposed along the $y$ direction at the boundaries of the grid $(y= \pm 7.5 \mu \mathrm{m}){ }^{73}$ To simulate the strong, uniform anchoring at the polyimide alignment layers used in the experiments, fixed boundary conditions for $\alpha$ at the top and bottom wall are defined such that the angle between $\mathbf{n}_{0}$ and the $x$-axis is $\phi[\alpha(y, z=5 \mu \mathrm{m})=$ $\alpha(y, z=-5 \mu \mathrm{m})=\phi]$. For comparison, two sets boundary conditions are used at the edges of the ray: (1) all four edges promote strong planar anchoring along the long-axis of the ray, or longitudinal anchoring $[\alpha(y, z= \pm h / 2)=0$, for $-w / 2 \leq$ $y \leq w / 2$ and $\alpha(y= \pm w / 2, z)=0$ for $-h / 2 \leq y \leq h / 2]$ as shown schematically in Fig. 5a and (2) longitudinal anchoring on the two vertical side walls of the ray $[\alpha(y= \pm w / 2, z)=0$ for $-h / 2 \leq$ $y \leq h / 2]$ and degenerate planar anchoring on the top and bottom horizontal surfaces of the ray, which is a more accurate representation of the boundary conditions in the experimental system (Fig. 5b).

Calculations are initiated using a uniform solution, $\alpha(y, z)=$ $\phi$, and a simple checkerboard updating scheme in which the local solution at every lattice point is replaced with the average of the four nearest neighbors was implemented to iteratively relax the solution. ${ }^{72}$ Roughly $2 \times 10^{4}$ iterations are needed to achieve $0.1 \%$ accuracy in the convergence of the elastic free energy per unit length $U_{\mathrm{el}} / L$. To monitor the convergence of the solution, every $10^{3}$ iterations, $U_{\mathrm{el}} / L$ is evaluated by numerical differentiation of $\alpha$, and subsequently integrated over the $y-z$ plane excluding the area of the ray. In Fig. $5 \mathrm{c}$ and d, false color plots of $\alpha(y, z)$ with $\phi=\pi / 2$ for both sets of boundary conditions are shown. Comparing the plots in Fig. $5 \mathrm{c}$ and d, a clear consequence of allowing for azimuthal degeneracy in the surface anchoring on top and bottom edges of the ray is that there are significantly less deformations of $\mathbf{n}(\mathbf{r})$ near those edges, whereas for the longitudinal case the $\mathbf{n}(\mathbf{r})$ distortions propagate in all directions away from the ray.
The elastic energy per unit length $U_{\mathrm{el}} / L$ as a function of $\phi$ is determined from solutions $\alpha(y, z)$ at various $\phi$ (Fig. 5e) with the average elastic constant $K=4 \mathrm{pN}$. For longitudinal anchoring, $U_{\mathrm{el}} / L$ (squares) is larger than $U_{\mathrm{el}} / L$ for degenerate anchoring boundary conditions (circles) at all $\phi>0$. Both are harmonic up to $\phi=\pi / 2$ in light of the high quality fits to a quadratic potential

$$
U_{\mathrm{el}} / L=2 \pi K(C / L) \phi^{2},
$$

where $C / L$ is the capacitance per unit length. Using $C / L$ as the only free parameter in the fits shown in Fig. 5e, we obtain $C / L \approx$ 0.181 and $C / L \approx 0.151$ for longitudinal anchoring and degenerate planar anchoring, respectively. To estimate the stiffness $k=2 \pi K N C$ of the orientational potential well for a 4-star, we use $C / L=0.151$ for a single ray, $N=4, L=3 \mu \mathrm{m}$, and find $k=2 \pi K N C$ $\approx 4.55 \times 10^{-17} \mathrm{~J} \mathrm{rad}^{-2}$. The discrepancy between $C / L$ for longitudinal anchoring and $C / L$ for degenerate planar anchoring could be larger if end effects and elastic interactions between the rays were taken into account. More sophisticated numerical methods in three dimensions, such as Landau-de Gennes free energy minimization ${ }^{67}$ or lattice Boltzmann simulations,${ }^{66}$ would be necessary to fully understand these effects.

\section{4 $\quad \mathrm{N}$-star elastic interactions}

At long range, the separation dependence of the elastic interactions between pairs of $N$-stars should follow from the symmetry of $\mathbf{n}(\mathbf{r})$ deformations surrounding each isolated particle. We test this by positioning pairs of $\mathrm{N}$-stars such that the attraction along their center-to-center separation vector $\mathbf{R}$ is a maximum. The optical tweezers are turned off and the motion of the $N$-star pair under elastic forces is determined by taking digital video. To provide contrast in the microscopy images, we place the sample between crossed polarizers and observe the bright birefringent textures from the rays having $\mathbf{n}(\mathbf{r})$ distortions on top of a dark background.
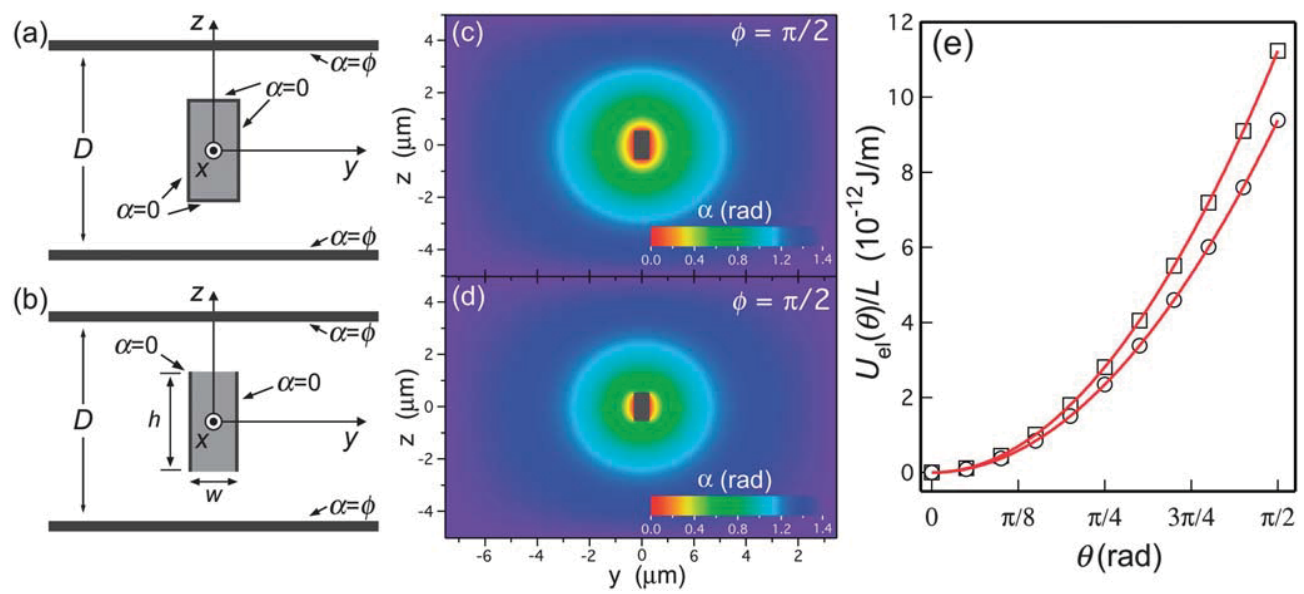

Fig. 5 Numerical solutions for $\mathbf{n}(\mathbf{r}$ ) around the rectangular cross-section ray of an $N$-star using two sets of boundary conditions on the rays edges (width $w=0.5 \mu m$ and height $h=1 \mu \mathrm{m}$ ). Cell gap $D=10 \mu \mathrm{m}$. (a) Longitudinal anchoring on all four edges of the ray, and (b) longitudinal anchoring on the two vertical edges of the ray and degenerate planar anchoring on the top and bottom edges. Strong anchoring is enforced at the top and bottom boundaries. (c and d) False color plots of results for the angle between $\mathbf{n}(\mathbf{r})$ and the $x$-axis, $\alpha(y, z)$, for the boundary conditions shown in (a) and (b), respectively. The angle between the ray and $\mathbf{n}_{0}, \phi=\pi / 2$, in both cases. (e) Elastic energy per unit length $U_{\mathrm{el}} / L$ as a function of $\phi$ for longitudinal anchoring (squares), and $U_{\mathrm{el}} / L$ for degenerate anchoring on the top and bottom edges (circles). Red curves show fit results using a harmonic $U_{\mathrm{el}} / L=2 \pi K(C / L) \phi^{2}$ with $K=4 \mathrm{pN}$. 
A pair of 3-stars having their elastic dipole moments $\mathbf{p}_{\mathrm{el}}$ parallel to $\mathbf{n}_{0}$ were positioned using optical tweezers such that their center-to-center separation vector $\mathbf{R}$ was also parallel to $\mathbf{n}_{0}$. This particular orientation of $\mathbf{R}$ relative to $\mathbf{p}_{\mathrm{el}}$ results in the strongest attraction under dipole-dipole forces. Their subsequent motion after being positioned and released was probed using video microscopy and particle tracking to determine both 3 -star's position in every frame. For each frame in a video, our tracking algorithm first thresholds the frames appropriately giving an accurate binary representation of the two $\mathrm{N}$-stars. The center of mass of the intensity distribution of each $N$-star is then determined to an accuracy of $\approx 0.5-1$ pixels $\approx 50-100 \mathrm{~nm}$, which is a small fraction of the lateral size of an $\mathrm{N}$-star.

Significant attractive forces between two 3-stars occur over large separations $R \approx 12 \mu \mathrm{m}$ (Fig. 6a), and they subsequently come in contact such that the ray parallel to $\mathbf{n}_{0}$ of one 3 -star touches the inner vertex opposite to the ray parallel to $\mathbf{n}_{0}$ of the second 3-star. We also observe a strong screening of the elastic force at separations greater than the cell gap $R>D \approx 10 \mu \mathrm{m}$ as has been measured for micron-sized spheres in thin cells. ${ }^{41}$ Interestingly, anti-parallel 3-stars that are aggregated such that one ray's tip contacts the end of the adjacent ray rotate their bodies significantly about $\mathbf{n}_{0}$ due to an effective short range repulsion between the rays (Fig. 6d), whereas parallel 3-stars tend to aggregate without as much out of plane twisting at short range (Fig. 6c). Presumably, this is because the neighboring rays on opposite 3-stars in Fig. 6c are attracted strongly to each other whereas for anti-parallel 3-stars there is a repulsion between the out of focus ray of the bottom 3-star and the upper 3-star's ray oriented parallel to $\mathbf{n}_{0}$.

In Fig. 6b, a plot of the time dependence of the interparticle separation $R(t)$ is shown for the two 3-stars in Fig. 6a. While the elastic force $F_{\text {el }}$ drives the two 3-stars towards each other, fluid flow due to their motion is Stokes-like with a corresponding Reynolds number that is small, Re $\sim \rho v L / \eta \sim 10^{-7}$ where the density of $5 \mathrm{CB}, \rho \approx 10^{3} \mathrm{~kg} \mathrm{~m}^{-3}$, and the highest velocities and viscosities in our experiments are of order $v \sim 1 \mu \mathrm{m} \mathrm{s}^{-1}$ and
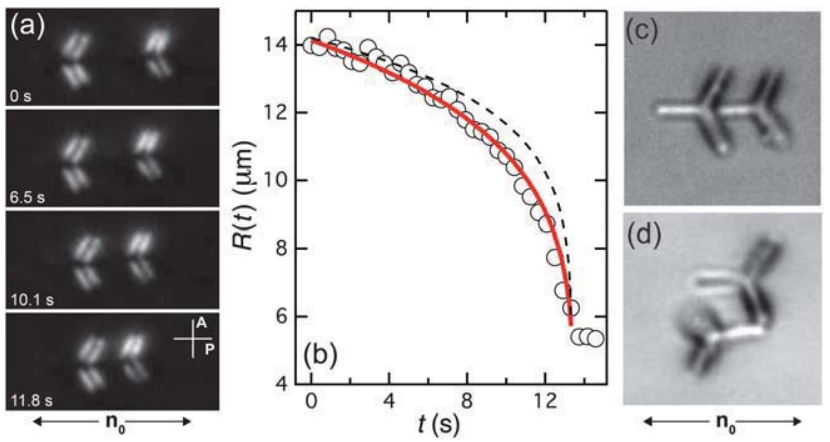

Fig. 6 (a) Series of video frames showing a pair of parallel, ground state 3-stars interacting under long-range elastic forces. (b) Data for center-to-center separation $R$ as a function of time for the 3-stars are shown. Red curve displays fit results for the expected trajectory of a pair of parallel dipoles and the dashed curve shows the fit results for a pair of attractive quadrupoles. (c) Image under parallel polarizers of the final state of the pair of 3-stars in (a). (d) Anti-parallel dipoles attract strongly when $\mathbf{R}$ is orthogonal to $\mathbf{n}_{0}$ and assemble in a highly twisted state. $\eta \sim 0.1$ Pa s. Thus, inertial forces are negligible, and the elastic force driving their motion, $F_{\mathrm{el}}$, is balanced by viscous drag, $F_{\mathrm{d}}=$ $\gamma \mathrm{d} R / \mathrm{d} t$, where $\gamma$ is the drag coefficient for a 3-star translating parallel to $\mathbf{n}_{0}$. In addition, the Erickson number, a dimensionless ratio of viscous to elastic forces, which describes the degree of coupling between the director field and hydrodynamic velocity field, is small ( $\operatorname{Er} \sim \eta v L / K \ll 1)$, and consequently, nonlinear drag effects due to coupling between $\mathbf{n}(\mathbf{r})$ and shear flows are also negligible. This is evident from the fact that the birefringent textures around the two 3-stars do not change outside of small thermal fluctuations even while they are traveling at the highest velocities $\approx 2 \mu \mathrm{m} \mathrm{s}^{-1}$ near contact (Fig. 6a). Moreover, estimating the Erickson number for a 3-star traveling at $2 \mu \mathrm{m}$ $\mathrm{s}^{-1}$ in $5 \mathrm{CB}$ we find $\mathrm{Er} \sim 10^{-2}$ where we have used the largest value of the three Miesowitz viscosities for $5 \mathrm{CB}, \eta \sim 0.1 \mathrm{~Pa} \mathrm{~s}, K=$ $4 \mathrm{pN}$, and $L=3 \mu \mathrm{m}$.

For $\mathbf{R} \| \mathbf{p}_{\mathrm{el}}$, elastic dipole-dipole forces are expected to decay with separation as a power-law: $F_{\mathrm{el}}=\kappa / R^{4}$, where $\kappa$ is a constant that depends on the elastic constants of $5 \mathrm{CB}$, the strength and type of surface anchoring, as well as the detailed geometry of the 3 -star. The equation of motion, $\kappa / R^{4}-\gamma \mathrm{d} R / \mathrm{d} t=0$, can be integrated analytically to arrive at the solution, $R(t)=\left(R_{0}{ }^{5}-5 A t\right)^{1 / 5}$, where $R_{0}=14.1 \mu \mathrm{m}$ is the separation between the two 3-stars at $t=0$ and the parameter $A=\kappa / \gamma$ is the ratio of $\kappa$ and the drag coefficient $\gamma$. A least squares fit using $A$ as the only adjustable parameter to the trajectory data yields $A \approx(8.4 \pm 0.2) \times 10^{3} \mu \mathrm{m}^{5}$ $\mathrm{s}^{-1}$ as shown with the red curve in Fig. $6 \mathrm{~b}$. For comparison, an estimate for $A$ can be obtained using dimensional analysis. In particular, $\kappa$ must be proportional to an elastic constant of $5 \mathrm{CB} K$ and a characteristic length $L$ to the fourth power, whereas the drag coefficient scales linearly with $L, \gamma \sim 2 \eta L$, and $\eta$ is a shear viscosity of $5 \mathrm{CB}$. Using the average elastic constant of $5 \mathrm{CB}, K=4 \mathrm{pN}$, the length of one ray of a 3-star $L \approx 3 \mu \mathrm{m}$, and the Miesowicz viscosity for shear flow predominantly parallel to $\mathbf{n}_{0} \eta \approx 0.04 \mathrm{~Pa} \mathrm{~s}$ at $T=$ $23{ }^{\circ} \mathrm{C},{ }^{78,79}$ we obtain $\kappa \sim 2 K L^{3} / \eta \approx 5.4 \times 10^{3} \mu \mathrm{m}^{5} \mathrm{~s}^{-1}$ which agrees qualitatively with the experimentally determined value for $A$. For general attractive power-law pair interaction forces, $F_{\mathrm{el}}=A_{n} / R^{n}$, where the exponent $n$ is a positive integer greater than 1 and $A_{n}$ is the amplitude, the solution to the equation of motion is $R(t)=$ $\left[R_{0}{ }^{n+1}-(n+1) A_{n} t / \gamma\right]^{1 /(n+1)}$. We are unable to fit the trajectory data as well as the case of $n=4$ using any other possible $n$, and, to demonstrate the larger discrepancy for $n \neq 4$, the fit results for a quadrupolar power-law with exponent $n=6$ are also shown in Fig. $6 \mathrm{~b}$ (dashed curve).

Two 4-stars are expected to interact at long-range via quadrupole-quadrupole forces, which provide the strongest attraction when $\mathbf{R}$ is oblique to $\mathbf{n}_{0}$. As shown in a series of video frames in Fig. 7a, two 4-stars move laterally and $R$ decreases while the angle between $\mathbf{R}$ and $\mathbf{n}_{0}$ approaches $\approx 42^{\circ}$. Over the duration of the last two frames displayed in Fig. 7a, the 4-stars attract and aggregate when the tips of adjacent rays contact. Data for $R(t)$ are shown in Fig. 7b along with fits to both a quadrupolar $n=6$ and dipolar $n=4$ trajectory. A fit to $R(t)=$ $\left(R_{0}{ }^{7}-7 A_{6} t\right)^{1 / 7}$ using $R_{0}=11.9 \mu \mathrm{m}$ gives $A_{6} \approx 1.4 \times 10^{5} \mu \mathrm{m}^{7} \mathrm{~s}^{-1}$. Again, using dimensional analysis to estimate the parameter $A_{6}$ yields $A_{6} \sim 4 K L^{5} / \eta \approx 10^{5} \mu \mathrm{m}^{7} \mathrm{~s}^{-1}$, which also agrees qualitatively with the measurement. The value we use for the shear 


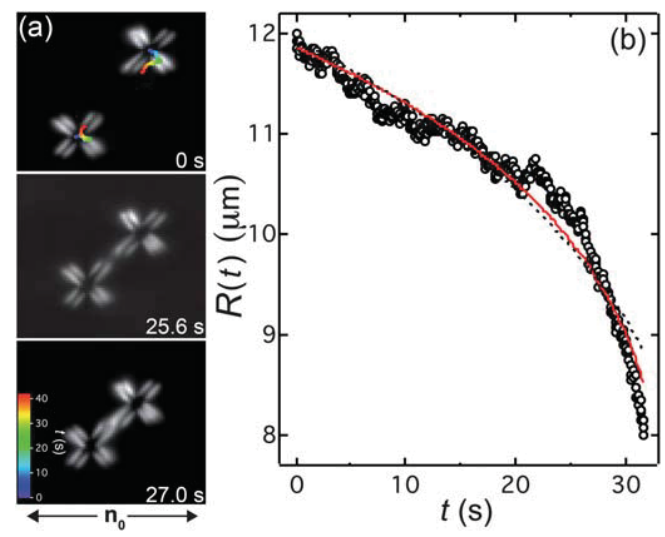

Fig. 7 (a) Three frames extracted from a video showing two 4-stars that are positioned such that their center-to-center separation vector $\mathbf{R}$ is at a 45 degree angle to $\mathbf{n}_{0}$. After release, they initially move laterally until $\mathbf{R}$ reaches a $42^{\circ}$ angle relative to $\mathbf{n}_{0}$. When $\mathbf{R}$ is at $\approx 42^{\circ}$ to $\mathbf{n}_{0}$, elastic attraction drives them together such that the tips of adjacent rays touch. Arrows illustrate the direction of the elastic force acting on each 4-star. (b) Data for $R(t)$ as a function of time (circles) determined with image analysis on the video shown in (a) when the angle between $\mathbf{R}$ and $\mathbf{n}_{0}$ is approximately $42^{\circ}$. A fit using the expected $R(t)$ for a quadrupole-quadrupole attraction is shown with the red curve. Dashed line shows the results of a fit using a dipole-dipole attractive interaction.

viscosity of $5 \mathrm{CB}, \eta \sim 0.04 \mathrm{~Pa}$ s, is most likely an under-estimate since the shear flow is predominantly along a $45^{\circ}$ angle to $\mathbf{n}_{0}$. At short-range, there are a number of possibilities for how 4-stars assemble together. For example, in the case of the two 4-stars shown in Fig. 7, the two aggregate with the tips of their rays touching while in other cases we observe aggregated states with adjacent rays interlocking.

In Fig. 8a, a series of video frames show two 5 -stars oriented such that $\mathbf{p}_{\mathrm{el}}$ is anti-parallel and $\mathbf{R}$ is approximately orthogonal to $\mathbf{n}_{0}$. After release from the optical trap, the two 5 -stars attract while moving laterally to one another resulting in the angle between $\mathbf{R}$ and $\mathbf{n}_{0}$ decreasing from $\approx 85^{\circ}$ to $\approx 74^{\circ}$ over the course of their motion. In Fig. $8 \mathrm{~b}$, data for the time dependence of $R(t)$ determined with image analysis is shown along with fits to the expected $R(t)$ for both a dipolar pair interaction (red curve) and quadrupolar interaction (dashed curve). The dipolar $R(t)$ fits the experimental data more reasonably than the quadrupolar case and gives a value for $A_{4} \approx 2350 \mu \mathrm{m}^{5} \mathrm{~s}^{-1}$ which is roughly a factor of 2 smaller than $A_{4}$ found for parallel ground state 3 -stars $\left(A_{4} \approx 5.4 \times 10^{3} \mu \mathrm{m}^{5} \mathrm{~s}^{-1}\right)$. This difference could arise from the fact that $\mathbf{p}_{\mathrm{el}}$ 's are antiparallel for the 5 -stars in Fig. 8 and parallel for the 3-stars in Fig. 6; however, the difference in shape between 3 -stars and 5-stars will result in different dipole moments and the drag coefficient is larger for 5-stars.

We have also studied the elastic interactions between similar $\mathrm{N}$-stars in different states (Fig. 9b and e) or different $\mathrm{N}$-stars in their respective ground states such as ground state quadrupolar 4-star and ground state dipolar 5-star (Fig. 9c and f). In the case of the metastable 5 -star interacting with a ground state 5 -star, the elastic dipole moments of the two particles are orthogonal, one parallel to $\mathbf{n}_{0}$ and the metastable $\mathbf{p}_{\mathrm{el}}$ orthogonal to $\mathbf{n}_{0}$, so the attractive force is largest when the relative position of the two dipoles are nearly $45^{\circ}$ to $\mathbf{n}_{0}$. They assemble in a state with their
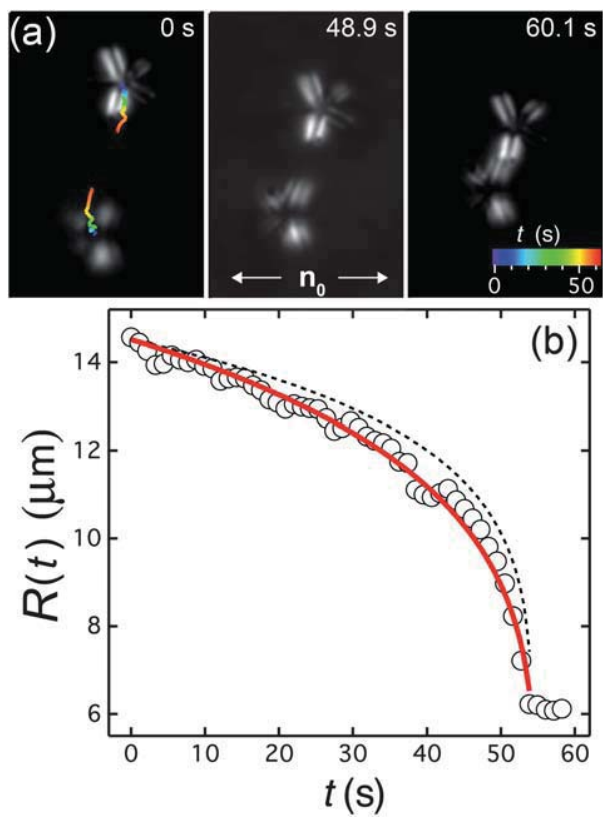

Fig. 8 (a) Series of frames extracted from a video showing a pair of anti-parallel 5 -stars positioned such that $\mathbf{R}$ is perpendicular to $\mathbf{n}_{0}$. After release, the 5-stars attract and assemble into a stable aggregate such that their adjacent rays contact end-to-end. (b) A plot of the time dependence of $R(t)$. Fits using the expected $R(\mathrm{t})$ for a dipolar interaction $\left(F_{\mathrm{el}} \sim 1 / R^{4}\right)$ and a quadrupolar interaction $\left(F_{\mathrm{el}} \sim 1 / R^{6}\right)$ are shown with the red and dashed curves, respectively.

rays interlocking and their center-of-mass positions closer $(R \approx$ $3.2 \mu \mathrm{m}$ ) than for other assemblies ( $R$ typically ranges from 6 to 7 $\mu \mathrm{m}$ after aggregation). For the quadrupolar 4-star and dipolar 5star having $\mathbf{p}_{\mathrm{el}} \| \mathbf{n}_{0}$, strong attraction occurs when $\mathbf{R}$ is at a $38^{-}$ $43^{\circ}$ angle to $\mathbf{n}_{0}$ and the two $N$-stars assemble with adjacent rays touching and shifted laterally by roughly the width of the ray (Fig. 9a and d). This position overlaps distorted regions near
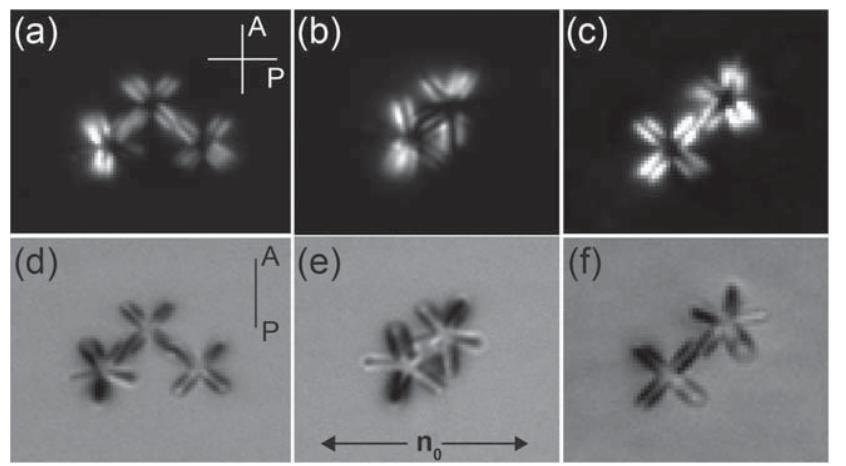

Fig. 9 Images of aggregated $\mathrm{N}$-stars made up of (a) a single 5-star and two 4star chain, (b) different states and equal $N$ : metastable and ground state 5-star, and (c) different ground state $\mathrm{N}$-stars: 4 - and 5-star. All three assemblies were formed by positioning the $N$-stars using optical tweezers such that the angle between $\mathbf{R}$ and $\mathbf{n}_{0}$ results in the largest elastic attraction at long-range. Within certain combinations of $\mathrm{N}$-star assemblies the rays of adjacent $\mathrm{N}$-stars interdigitate near contact such as the case of a ground and metastable state 5 -star ( $b$ and e). Often the tips of adjacent rays contact as for a 4- and 5-star in their respective ground states as shown in (c and $f$ ). 
their rays, which alleviates the deformations lowering the overall elastic energy. Interestingly, at long-range, a ground state 5-star approaches a two 4-star chain at an oblique angle of $\approx 30-35^{\circ}$ and attracts to a selective ray of the chain at nearly a $45^{\circ}$ angle at short-range $(R<8 \mu \mathrm{m})$.

\section{Conclusions}

We have found that concave star-shaped colloids exhibit very different behavior than convex polygonal colloids when suspended in uniformly aligned NLCs. Micron-scale $N$-star colloids having anisotropic, rod-like protrusions, orient their body axes relative to the alignment direction of a NLC in a manner that reflects the rotational and mirror symmetries of their geometries, bulk elasticity, and the interfacial anchoring properties of the NLC on their surfaces. In particular, concave $N$-stars having odd- $N$ such as 3-stars and 5-stars form elastic dipoles with the dipole moment parallel to the far-field director in equilibrium whereas convex 3- and 5-sided polygons induce elastic dipoles perpendicular to the director.

In addition to an equilibrium ground state corresponding to the smallest overall elastic energy, a given $N$-star can also be trapped at a different orientation, corresponding to a metastable configuration having higher elastic energy. Whereas such metastable states are readily observed for concave $\mathrm{N}$-star colloids, they have not been observed for dispersions of convex regular polygonal platelets prepared in a similar fashion. Evidently, the slender rays of $\mathrm{N}$-stars facilitate metastable configurations and either the metastable states of regular polygonal platelets are too unstable to be observed at the aspect ratios $(\approx 3-5)$ studied in ref. 15 or the energy of the metastable state is much larger than the ground state.

We have characterized the relative elastic energies between the observed ground and metastable configurations using a simple model in which the total energy is approximated as a superposition of the known energy for rod-like inclusions held at appropriate orientations. Moreover, the model allows for estimating the energy cost for rotations of $\mathrm{N}$-stars away from the global minimum within the ground state as well as the local minimum within the metastable configuration. Although elastic deformations resist a transition to the ground state through a body rotation, an additional topological barrier exists which stabilizes the metastable configuration. Experiments that probe the orientational fluctuations within a local minimum of the orientational potential well for various $\mathrm{N}$-stars may provide a micro-scale measurement of the numerous shear-viscosities of NLCs since the flow fields for rotating high-aspect ratio rods are known. ${ }^{74,75}$

Future experiments and calculations could probe how the relative energies of the metastable and ground state configurations depend upon aspect ratio of the individual rays. For instance, changing the shape of the rays from prolate to oblate would result in a geometry similar to that of a regular convex polygon (at some particular aspect ratio) allowing one to tune between a concave $N$-star shape and a convex polygon shape using one geometrical parameter. For oblate rays, the outer convex vertices would play a greater role in the overall elastic energy, and if the aspect ratio is high enough, the ground state should switch to a $\mathbf{n}(\mathbf{r})$-configuration having $\mathbf{p}_{\mathrm{el}} \perp \mathbf{n}_{0}$ similar to convex polygons, since in the limit of large oblate aspect ratio the particle shape would essentially be a regular polygon.

Beyond isolated $N$-stars, we have also studied interactions between pairs of $\mathrm{N}$-stars using a combination of optical tweezers and video microscopy. We have found that the mirror symmetry of $\mathbf{n}(\mathbf{r})$ is sufficient to predict the dominant elastic power-law pair interaction at long range. At short-range, depending upon the symmetry of the $\mathrm{N}$-stars, the ray of one $\mathrm{N}$-star tends to interdigitate between the rays of an adjacent $N$-star, and penetrate to the nearby core for $N=3$, yet not as readily for $N=4$ or 5 . In the case of two anti-parallel 3-stars that aggregate tip-to-tip, near-field elasticity drives out-of-plane rotations, minimizing the distortion of the nematic director field, whereas parallel 3-stars assemble tipto-core without significant rotations at short-range. $\mathrm{N}$-star assemblies having more than two particles can display various history-dependent low-energy configurations in which adjacent rays may possibly interlock. In order to understand how these short-range effects influence the elastic energy of such complex assemblies, more sophisticated three-dimensional modeling is needed. Lastly, we anticipate that driving N-stars in NLCs using applied fields could result in interesting hydrodynamic effects, such as dynamic lift, ${ }^{76}$ or elastic and dielectric effects such as torques induced during a Fréedericksz transition. ${ }^{77}$

\section{Acknowledgements}

C. P. L. acknowledges support provided by ICAM Branch Contributions to begin this research and acknowledges financial support from the Swiss State Secretariat for Education, Research and Innovation, the Adolphe Merkle Foundation and current funding from the Swiss National Science Foundation under the Ambizione Program (SNF Project - 142571).

\section{References}

1 C. C. Ho, A. Keller, J. A. Odell and R. H. Ottewill, Colloid Polym. Sci., 1993, 271, 469.

2 A. B. D. Brown, C. G. Smith and A. R. Rennie, Phys. Rev. E., 2000, 62, 951.

3 N. Bowden, F. Arias, T. Deng and G. M. Whitesides, Langmuir, 2001, 17, 1757.

4 J. H. Moon, A. J. Kim, J. C. Crocker and S. Yang, Adv. Mat., 2007, 19, 2508.

5 J. P. Rolland, B. W. Maynor, L. E. Euliss, A. E. Exner, G. M. Denison and J. M. DeSimone, J. Am. Chem. Soc., 2005, 127, 10096.

6 D. Dendukuri, D. C. Pregibon, J. Collins, T. A. Hatton and P. S. Doyle, Nat. Mater., 2006, 5, 365.

7 C. J. Hernandez and T. G. Mason, J. Phys. Chem. C, 2007, 111, 4474.

8 S. Badaire, C. Cottin-Bizonne, J. W. Woody, A. Yang and A. D. Stroock, J. Am. Chem. Soc., 2007, 129, 40.

9 S. C. Glotzer and M. J. Solomon, Nat. Mater., 2007, 6, 557.

10 K. J. Stebe, E. Lewandowski and M. Ghosh, Science, 2009, 325, 159. 
11 S. Sacanna and D. J. Pine, Curr. Opin. Colloid Interface Sci., 2011, 16, 96.

12 T. G. Mason, Phys. Rev. E, 2002, 66, 060402.

13 K. Zhao and T. G. Mason, Phys. Rev. Lett., 2007, 99, 268301.

14 S. Sacanna, W. T. M. Irvine, P. M. Chaikin and D. J. Pine, Nature, 2010, 464, 575.

15 C. P. Lapointe, T. G. Mason and I. I. Smalyukh, Science, 2009, 326, 1083.

16 K. Zhao and T. G. Mason, J. Am. Chem. Soc., 2012, 134, 18125.

17 K. Zhao, R. Bruinsma and T. G. Mason, Nat. Commun., 2012, 3, 801.

18 K. Zhao, R. Bruinsma and T. G. Mason, Proc. Natl. Acad. Sci. U. S. A., 2011, 108, 2684.

19 S. Sacanna, D. J. Pine and G.-R. Yi, Soft Matter, 2013, DOI: 10.1039/c3sm50500f.

20 P. Pieransky, Phys. Rev. Lett., 1980, 45, 569.

21 B. J. Park and E. M. Furst, Soft Matter, 2010, 6, 485.

22 J.-C. Loudet, A. G. Yodh and B. Pouligny, Phys. Rev. Lett., 2006, 97, 018304.

23 J.-C. Loudet and B. Pouligny, Europhys. Lett., 2009, 85, 28003.

24 E. P. Lewandowski, M. Cavallaro Jr, L. Botto, J. C. Bernate, V. Garbin and K. J. Stebe, Langmuir, 2010, 26, 15142.

25 P. Poulin, H. Stark, T. C. Lubensky and D. A. Weitz, Science, 1997, 275, 1770.

26 R. W. Ruhwandl and E. M. Terentjev, Phys. Rev. E, 1997, 55, 2958.

27 P. Poulin, V. Cabuil and D. A. Weitz, Phys. Rev. Lett., 1997, 79, 4862.

28 P. Poulin and D. A. Weitz, Phys. Rev. E, 1998, 57, 626.

29 Y. Gu and N. L. Abbott, Phys. Rev. Lett., 2000, 85, 4719.

30 H. Stark, Phys. Rep., 2001, 351, 387.

31 T. Yamamoto, J. Yamamoto, B. I. Lev and H. Yokoyama, Appl. Phys. Lett., 2002, 81, 2187.

32 O. Guzmán, E. B. Kim, S. Grollau, N. L. Abbott and J. J. de Pablo, Phys. Rev. Lett., 2003, 91, 235507.

33 M. Yada, J. Yamamoto and H. Yokoyama, Phys. Rev. Lett., 2004, 92, 185501.

34 J. Fukuda, H. Stark, M. Yoneya and H. Yokoyama, Phys. Rev. E, 2004, 69, 041706.

35 I. I. Smalyukh, O. D. Lavrentovich, A. N. Kuzmin, A. V. Kachynski and P. N. Prasad, Phys. Rev. Lett., 2005, 95, 157801.

36 I. Muševič, M. Škarabot, U. Tkalec, M. Ravnik and S. Žumer, Science, 2006, 313, 954.

37 C. Völtz, Y. Maeda, Y. Tabe and H. Yokoyama, Phys. Rev. Lett., 2006, 97, 227801.

38 J. Kotar, M. Vilfan, N. Osterman, D. Babič, M. Čopič and I. Pobera, Phys. Rev. Lett., 2006, 96, 207801.

39 O. P. Pishnyak, S. Tang, J. R. Kelly, S. V. Shiyanovskii and O. D. Lavrentovich, Phys. Rev. Lett., 2007, 99, 127802.

40 U. Ognysta, A. Nych, V. Nazarenko, I. Muševič, M. Škarabot, M. Ravnik, S. Žumer, I. Poberaj and D. Babič, Phys. Rev. Lett., 2008, 100, 217803.

41 M. Vilfan, N. Osterman, M. Copic, M. Ravnik, S. Zumer, J. Kotar, D. Babič and I. Poberaj, Phys. Rev. Lett., 2008, 101, 237801.
42 U. Ognysta, A. Nych, V. Nazarenko, M. Škarabot and I. Muševič, Langmuir, 2009, 25, 12092.

43 S. K. Pal, A. Agarwal and N. L. Abbott, Small, 2009, 5, 2589.

44 O. D. Lavrentovich, I. Lazo and O. P. Pishnyak, Nature, 2010, 467, 947.

45 U. M. Ognysta, A. B. Nych, V. A. Uzunova, V. M. Pergamenschik, V. G. Nazarenko, M. Škarabot and I. Muševič, Phys. Rev. E, 2011, 83, 041709.

46 F. Brochard and P. G. de Gennes, J. Phys. (Paris), 1970, 31, 691.

47 P. G. de Gennes, The Physics of Liquid Crystals, 1974, Claredon Press, Oxford, 1st edn, 1975.

48 In ref. 47, de Gennes provided an example problem that considers an arbitrarily shaped inclusion immersed in an aligned NLC, and argued that the lowest order monopole-like term, which decays with distance $R$ from the source as $\sim 1 / R$, cannot exist without an external torque acting on the object.

49 J. B. Fournier, Eur. J. Phys., 1993, 14, 184.

50 T. C. Lubensky, D. Pettey, N. Currier and H. Stark, Phys. Rev. E, 1997, 57, 610.

51 B. I. Lev, S. B. Chernyshuk, P. M. Tomchuk and H. Yokoyama, Phys. Rev. E, 2002, 65, 021709.

52 F. R. Hung, Phys. Rev. E: Stat., Nonlinear, Soft Matter Phys., 2009, 79, 021705.

53 V. M. Pergamenshchik and V. A. Uzunova, Condens. Matter Phys., 2010, 13, 33602.

54 V. M. Pergamenshchik and V. A. Uzunova, Phys. Rev. E, 2011, 83, 021701.

55 C. Lapointe, A. Hultgren, D. M. Silevitch, E. J. Felton, D. H. Reich and R. L. Leheny, Science, 2004, 303, 652.

56 C. Lapointe, N. Cappallo, D. H. Reich and R. L. Leheny, J. Appl. Phys., 2005, 97, 10Q304.

57 U. Tkalec, M. Škarabot and I. Muševič, Soft Matter, 2008, 4, 2402.

58 F. Mondiot, S. Prathap Chandran, O. Mondain-Monval and J.-C. Loudet, Phys. Rev. Lett., 2009, 103, 238303.

59 J. B. Rovner, D. S. Borgnia, D. H. Reich and R. L. Leheny, Phys. Rev. E, 2012, 86, 041702.

60 J. Deschamps, J. P. M. Trusler and G. Jackson, J. Phys. Chem. B, 2008, 112, 3918.

61 I. I. Smalyukh, A. V. Kachynski, A. N. Kuzmin and P. N. Prasad, Proc. Natl. Acad. Sci. U. S. A., 2006, 103, 18048.

62 I. Muševič, M. Škarabot, D. Babic, N. Osterman, I. Poberaj, V. Nazarenko and A. Nych, Phys. Rev. Lett., 2004, 93, 187801.

63 M. Škarabot, M. Ravnik, D. Babič, N. Osterman, I. Poberaj, S. Žumer, I. Muševič, A. Nych, U. Ognysta and V. Nazarenko, Phys. Rev. E, 2006, 73, 021705.

64 S. D. Durbin, S. M. Arakelian and Y. R. Shen, Phys. Rev. Lett., 1981, 47, 1411.

65 N. V. Madhusudana and R. Pratibha, Mol. Cryst. Liq. Cryst., 1982, 89, 249.

66 C. J. Smith and C. Denniston, J. Appl. Phys., 2007, 101, 014305.

67 M. Ravnik and S. Zumer, Liq. Cryst., 2009, 36, 1201.

68 F. Alouges and B. D. Coleman, J. Phys. A: Math. Gen., 1999, 32, 1177.

69 S. V. Burylov and Y. L. Raikher, Phys. Lett. A, 1990, 149, 279. 70 S. V. Burylov and Y. L. Raikher, Phys. Rev. E, 1994, 50, 358. 
71 D. Andrienko, M. P. Allen, G. Skacej and S. Zumer, Phys. Rev. E, 2002, 65, 041702.

72 W. H. Press, S. A. Teukolsky, W. T. Vetterling and B. P. Flannery, Numerical Recipes in $C$, Cambridge University Press, 2nd edn, 1992.

73 The presence of finite size effects were determined by doubling the lateral size of the grid and calculating $\alpha(y, z)$ in the same fashion yielding a total energy at fixed $\phi$ different from the 300 by 200 case by less than $0.1 \%$.
74 Z. Cheng, P. M. Chaikin and T. G. Mason, Phys. Rev. Lett., 2002, 89, 108303.

75 T. M. Squires and T. G. Mason, Rheol. Acta, 2010, 49, 1165. 76 J. B. Rovner, C. P. Lapointe, D. H. Reich and R. L. Leheny, Phys. Rev. Lett., 2010, 105, 228301.

77 C. P. Lapointe, S. Hopkins, T. G. Mason and I. I. Smalyukh, Phys. Rev. Lett., 2010, 105, 178301.

78 A. G. Chmielewski, Mol. Cryst. Liq. Cryst., 1986, 132, 339.

79 M. Cui and J. R. Kelly, Mol. Cryst. Liq. Cryst., 1999, 331, 49. 